\title{
Balkanologie
}

Balkanologie Revue d'études pluridisciplinaires

Vol. IV, $n^{\circ} 1 \mid 2000$

Volume IV Numéro 1

\section{Le groupe de Prague : le cinéma en tant que production culturelle non-nationaliste dans la Yougoslavie des années 1980}

Jasmina Papa

\section{CpenEdition}

Journals

Édition électronique

URL : https://journals.openedition.org/balkanologie/776

DOI : 10.4000/balkanologie.776

ISSN : 1965-0582

Éditeur

Association française d'études sur les Balkans (Afebalk)

Édition imprimée

Date de publication : 1 septembre 2000

ISSN : 1279-7952

Référence électronique

Jasmina Papa, « Le groupe de Prague : le cinéma en tant que production culturelle non-nationaliste dans la Yougoslavie des années 1980 », Balkanologie [En ligne], Vol. IV, $n^{\circ} 1$ | 2000, mis en ligne le 29 juillet 2010, consulté le 28 juin 2022. URL : http://journals.openedition.org/balkanologie/776 ; DOI : https://doi.org/10.4000/balkanologie.776

Ce document a été généré automatiquement le 17 décembre 2020.

(C) Tous droits réservés 


\title{
Le groupe de Prague : le cinéma en tant que production culturelle non- nationaliste dans la Yougoslavie des années 1980
}

\author{
Jasmina Papa
}

1 Cet article présente les membres d'un groupe de réalisateurs, au fondement de la renaissance du cinéma yougoslave à la fin des années 1970 et dans les années 1980, à une époque de crise et de fractionnalisation au sein même de la fédération yougoslave. Le groupe des six réalisateurs (Rajko Grlić, Srđan Karanović, Emir Kusturica, Goran Marković, Goran Paskaljević, et Lordan Zafranović) est connu sous le nom de "Groupe de Prague", du fait qu'ils aient tous suivi des études d'art à Prague. Ce groupe a connu un succès populaire et une critique positive pour leurs films qui étaient des énoncés politiques et esthétiques. Ces réalisateurs, bien que ne célébrant certainement pas les idéaux yougoslaves tels qu'ils existaient à l'époque, ne se sont pas laissés entrainer par les mouvements nationalistes au contraire de nombreux autres artistes, notamment les poètes, écrivains et rockers. Cet article cherche à mettre en évidence les principaux thèmes de leurs travaux, à comprendre et expliquer leur importance et souligner leurs rapports épineux avec les politiques de la fédération et de la formation identitaire républicaine.

2 La politique vis-à-vis de la réalisation cinématographique dans la Yougoslavie de l'après Deuxième Guerre mondiale était obscure mais, en de nombreuses façons, en relation avec des politiques plus vastes et le développement des mass-medias ${ }^{1}$. Le développement de l'autogestion socialiste à la suite de la rupture avec Staline et l'expulsion du Kominform en 1948 n'a pas donné lieu à une libéralisation immédiate de la réalisation cinématographique ni non plus des mass-medias. Ceci était dû, en partie, à la continuation de la crise, aussi bien politique qu'économique, et au besoin d'un nouvel effort dans la légitimation du système politique. Le retrait soudain des subventions étatiques au cinéma au début des années 1950 n'a pas été réalisé avec un 
soutien inconditionnel. On peut néamoins l'analyser, en termes bourdieusiens, comme l'institution de la réalisation cinématographique en tant que champ culturel, dans lequel les réalisateurs ont à jouer un rôle clef. Lorsque les conditions politiques se sont orientées vers la libéralisation (initiée au début des années 1960, elle s'est accélérée en 1966 lorsqu'Aleksandar Ranković, un des plus grands conservateurs du Parti, a été écarté de la scène politique), elles ont donné lieu à une explosion de films critiques, appelé "Nouveau cinéma". La réaction contre le Nouveau cinéma fut essentiellement politique et n'était pas sans rapport avec les incessantes crises qu'a connues l'État fédéral entre 1968 et 1971.

3 Dans les années 1970, du fait de la réaction contre le Nouveau cinéma, moins de films furent réalisés, la plupart consistant en épopées coûteuses présentant une histoire noncritique du rôle de Tito et des Partisans dans la libération de la Yougoslavie. Toutefois, une mainmise générale sur les différents mass-médias était de plus en plus difficile à maintenir, suggérant qu'une fois constitué, un champ culturel est difficile à contrôler sans que l'État ne recoure explicitement à la violence. Au contraire, la constitution de 1974 attribua des pouvoirs accrus aux Républiques et créa une situation dans laquelle la diversité culturelle et l'expérimentation continua de prospérer, d'autant plus que la censure des productions cinématographiques devenait de plus en plus dure à mettre en œuvre.

4 La complexité s'est accrue dans les années 1980, la récession économique coïncidant avec une fractionnalisation politique générale ${ }^{2}$. La situation formelle, légale, était bien moins importante que la pratique effective dans la détermination de ce qui se déroulait dans les mass-medias et les films. Le fait que, dans une période de crise économique, la réalisation cinématographique était toujours perçue comme une part importante de la production culturelle signifiait que le financement des films provenait de plus en plus de sources de plus en plus variées, allant des républiques à, plus tard, des coopérations étrangères. Ceci aida les réalisateurs du Groupe de Prague, établis en tant que réalisateurs talentueux aussi bien dans qu'en dehors du pays, à obtenir des financements pour tout film qu'ils souhaitaient réaliser, quoique dans le cadre d'un système administratif lourd.

\section{Les antécédents du Groupe de Prague}

5 Deux grands courants de réalisateurs de la période précédent le Groupe de Prague, c'est-à-dire entre la moitié des années 1960 et le début des années 1970, sont cruciaux en tant que précurseurs et créateurs. Ces courants sont le Nouveau cinéma yougoslave et la Nouvelle vague tchécoslovaque, incluant l'école des réalisateurs de Prague (FAMU). Ils ont grandement influencé l'esthétisme et l'esprit politique des réalisateurs du Groupe de Prague. Ces derniers, tout comme le Nouveau cinéma et la Nouvelle vague tchécoslovaque, s'intéressaient plus aux problèmes liés au socialisme qu'à ceux dérivant de la question nationale et du nationalisme. Ces deux mouvements sont apparus à une période de profonds changements politiques et sociaux, ce qui a également eu son impact sur le Groupe de Prague. Plus important peut-être, la réaction à ces deux mouvements, la censure de nombreux films et l'arrêt de la carrière de nombreux réalisateurs ont laissé leurs marques dans l'appréciation de ce qui peut se produire dans les sociétés autoritaires lorsque les limites, aux yeux de ceux qui détiennent le pouvoir, sont franchies. 


\section{Le nouveau cinéma yougoslave}

6 L'une des principales influences du Groupe de Prague est le Nouveau cinéma (Novi Film) en Yougoslavie, un mouvement artistique datant de la moitié des années 1960 - début des années 1970. Les membres les plus éminents du Nouveau film étaient : Aleksandar Petrovi\}, Bostjan Hladnik, Živojin Pavlović, Kokan Rakonjac, Marko Babac, Puriša lorđević, Dušan Makavejev, Krsto Papić, Zvonimir Žilnik, Vatroslav Mimica et Zvonimir Berković. Ils ont exercé deux types d'influence. La première est la sélection des sujets et leur présentation stylistique. La deuxième est le traitement provocateur de thèmes explicitement politiques. Les thèmes contemporains ou du passé récent remettaient en question des tabous généralement acceptés et révélaient les dysfonctions du système socialiste. Ces thèmes seraient à nouveau présents dans les travaux du Groupe de Prague.

7 La distinction majeure entre les deux groupes de réalisateurs réside dans leur perception des limites de la critique. Les membres du Nouveau cinéma affrontaient ouvertement le système, tête en avant. Au contraire, les membres du Groupe de Prague étaient moins explicites, plus circonspects et, pour cette raison peut-être, plus sages dans leurs critiques. D. Makavejev, le plus célèbre des réalisateurs du Nouveau cinéma, a rendu compte de cette différence lorsqu'il a labellisé le Groupe de Prague de "réalisateurs émasculés " ${ }^{3}$. Il est toutefois fort probable que ce soit sur les bases de la répression dont les réalisateurs du Nouveau cinéma ont été victimes que le Groupe de Prague ait tiré ses conclusions sur les limites du tolérable. En ce sens, l'influence du Nouveau cinéma sur le Groupe de Prague est moins directe, et constitue plus une leçon acquise sur comment ne pas s'opposer trop ouvertement au système.

On peut voir le développement du Nouveau cinéma comme une réflexion cinématographique spécifique sur de vastes problèmes culturels et politiques. Puisque l'"esthétisme socialiste" vint à l'emporter sur le "réalisme socialiste", le contrôle direct sur l'art prit moins d'ampleur. Il s'exprimait plus en définissant ce qui ne devait pas être montré qu'en imposant ce qui le devait. La lutte de pouvoir obscure et aléatoire entre les libéraux et les conservateurs signifiait qu'il existait, à certains moments, un espace pour une pression accentuée au nom de "motivations" autoritaires dans le cadre d'un point de vue non traditionnel. C'était en rupture radicale d'avec les précédentes périodes de l'art cinématographique où les réalisateurs devaient suivre au pied de la lettre les consignes émises par l'idéologie officielle et disposaient de peu de place pour $\mathrm{y}$ apposer leur touche personnelle.

9 Pour que les réalisateurs du Nouveau cinéma aient eu le droit de traiter de sujets originaux, dans le style dans lequel ils l'ont fait, des préconditions structurelles étaient requises. Non seulement il devait y avoir un haut niveau d'instruction cinématographique au sein des structures dominantes et au-delà, mais également un espace politique, parmi les réalisateurs, le public et les critiques pour dépeindre la réalité dans des formes alternatives ${ }^{4}$. Ainsi que l'ont synthétisé les Liehm, les films constituaient des représentations hautement personnelles, requérant une interprétation subjective de la vie, des individus et de la société, et une volonté d'appréhender les métaphores explicites que ces films présentaient ${ }^{5}$.

10 C'était le sommet du cinéma d'auteur du film yougoslave, de plus en plus caractérisé par la conscience, l'originalité et l'exploration critiques des réalisateurs des 
événements contemporains. C'est ce mélange qui les a rendus impopulaires auprès des autorités. Quand les mouvements de libéralisation ont cédé la place à des attitudes plus conservatrices envers l'art, des voix plus nombreuses se sont faites entendre pour réclamer de la "discipline”. Ainsi que Makavejev l'a exprimé : «l'Art, vous savez, est un terrain de jeux apprécié des conservateurs à l'encontre des libéraux, parce qu'ils peuvent parler de la morale, plutôt que de l'Art $»^{6}$. Le résultat fut que de nombreux réalisateurs furent publiquement critiqués, démis de leurs fonctions (ils étaient souvent enseignants d'art cinématographique à l'Université), et exclus du Parti. Leurs travaux furent, bien évidemment, interdits, parfois rétrospectivement.

11 Les principaux desseins du Nouveau cinéma semblent avoir été : tout d'abord, une libéralisation de la réalisation des contraintes bureaucratiques et des dogmes idéologiques; ensuite, la promotion d'expérimentation du film en tant que langage audiovisuel, à la suite des Nouvelles vagues française et tchécoslovaque; et, enfin, l'utilisation du film pour analyser des thèmes contemporains, de manière critique si nécessaire ${ }^{7}$. Ce mouvement cinématographique a coïncidé avec, et a reflété, une lutte politique plus globale qui s'est résolue en 1966 en faveur du courant libéral. Son symbole a été l'expulsion d'Aleksandar Ranković, qui a eu pour conséquence l'instauration d'un certain degré de démocratisation sociale et économique en Yougoslavie ${ }^{8}$. Dans le monde des réalisateurs, les membres du Nouveau cinéma ont ressenti avoir réalisé une importante victoire, et, effectivement, des films ont commencé à être réalisés qui "n'auraient pas pu l'être quand Ranković était au pouvoir $»^{9}$.

12 Dans des interviews récents, Makavejev a réfléchi sur cette période, constatant simplement : « la Yougoslavie était ainsi. Chacun a appris à créer son propre nid. Dans le système de Tito, il y avait de la place pour tout le monde $»^{10}$. Cela semble être un regard plutôt positif sur la période en question, d'autant plus qu'à la fin des années 1960, Makavejev a violemment ressenti le retour des politiques conservatrices, ayant donné lieu à des répressions dont l'intensité variait, à l'encontre de tous ceux qui ne se conformaient pas à la nouvelle orthodoxie. Le quotidien Borba, publié à Belgrade, et voix du régime, fut le premier à soulever la question des films en tant qu'hérésie antisocialiste dans un article intitulé "La vague noire dans notre cinéma ", publié en $1969^{11}$. À sa suite, articles et discours se sont succédés, signés des membres les plus importants aussi bien de la LCY (Ligue des Communistes de Yougoslavie) que de la JNA (Jugoslovenska Narodna Armija - Armée Populaire Yougoslave). La condamnation du Nouveau cinéma fut bien plus explicite dans les colonnes de Komunist, qui réitérait l'importance du cinéma en tant qu'activité publique et sociale et non comme un nouveau genre de jeu privé dépourvu de toute obligation sociale pour ceux qui y étaient impliqués ${ }^{12}$. Différents clans de la LCY percevaient le Nouveau cinéma comme délivrant à la population une message de faiblesse, doute et désespoir. De plus, « en parallèle avec cette note profondément anti-humaniste, certains n'ont pas hésité, surtout récemment, à déformer la réalité historique de notre vie quotidienne $»^{13}$.

13 Les conséquences de ces attaques furent graves. Makavejev fut expulsé du Parti et s'exila en France, WR: mystères de l'organisme fut interdit, et ne fut pas diffusé en Yougoslavie avant les années 1980. En 1973, un étudiant de la Faculté d'art cinématographique à Belgrade, Lazar Stojanović, fut condamné à 3 ans d'emprisonnement avec travaux forcés pour avoir réalisé un film intitulé Jésus plastique (Plastični Isus, 1971) pour valider son diplôme universitaire. Le film n'a été connu 
qu'après que Stojanović eut exprimé des avis politiques dissidents au sein de l'armée. Les officiers apprirent l'existence du film et le visionnèrent ${ }^{14}$. Aleksandar Petrović, qui avait bien noté le film, fut démis de ses fonctions au sein de la faculté, de même que nombre d'autres enseignants. La Cour Suprême serbe jugea que le film, mêlant des parades fascistes à des scènes pornographiques, était également offensant dans son portrait de Tito et constituait une « diversion politique » qui « ne pouvait être défendue par l'invocation de la liberté d'expression artistique $»^{15}$. C'était la première fois dans l'histoire yougoslave qu'un réalisateur était puni d'une peine de prison pour son travail.

\section{La FAMU et la Nouvelle vague tchécoslovaque}

14 La deuxième influence majeure des membres du Groupe de Prague est expressément révélée par leur dénomination. Le groupe a été profondément marqué par la Nouvelle Vague tchécoslovaque de réalisateurs qui a émergé en même temps que le Nouveau cinéma yougoslave et a été associée avec le mouvement politique de réforme connu sous le nom de "Printemps de Prague". De plus, tous les jeunes réalisateurs yougoslaves ont étudié à l'école d'art cinématographique de Prague (FAMU). De 1967 à 1971, cinq des six membres du Groupe de Prague (Karanović, Paskaljević, Marković, Grlić et Zafranović) ont fait leurs études ensemble, à l'époque où se sont déroulés les événements politiques et culturels les plus dramatiques, avec la floraison des réformes démocratiques et des expérimentations, et leur écrasement par l'invasion soviétique de 1968, suivi d'une période encore plus dure de répression, ironiquement appelée ultérieurement "normalisation". Il ne faut pas beaucoup d'imagination pour apprécier l'effet que pouvaient avoir ces événements extraordinaires sur un groupe d'étudiants yougoslaves de moins de 24 ans quand les tanks sont entrés.

15 Un certain nombre de réformes ont été prises par les communistes tchécoslovaques afin de réaliser la finalité du "socialisme à visage humain". L'une d'elles, initiée en 1963, à la même époque que les réformes sur le cinéma yougoslave, portait sur la réorganisation de la production cinématographique. Elle permettait, selon les Liehm, aux films d'être vus comme une forme d'art et, plus important encore, le développement d'une pluralité de talents et de styles hétérogènes ${ }^{16}$. Au contraire de la Yougoslavie, ce n'était pas seulement la réalisation cinématographique, mais tous les champs de la culture qui ont connu cette renaissance. En fait, le groupe d'écrivains tchèques qui a émergé à cette époque a grandement contribué à l'élaboration de cette diversité et de cette créativité dans le cinéma, notamment Bohumil Hrabal, Milan Kundera et Josef Skvorecky ${ }^{17}$.

16 À cette époque, la FAMU était le centre névralgique de la Nouvelle Vague tchécoslovaque - la plupart des principaux réalisateurs y avaient eux même étudié et beaucoup y enseignaient. Les étudiants étaient, par conséquent, en contact direct avec les principaux réalisateurs et écrivains de l'époque, et nombre d'entre eux leur prodiguaient des cours, et ce à une époque de grands bouleversements sociaux. S'appuyant sur le témoignage d'un étudiant qui fut diplomé au milieu des années 1960, Hames note que la faculté se trouvait dans un seul bâtiment, les étudiants, les professeurs et l'administration n'excédant pas 100 personnes, de telle sorte que «tout film terminé donnait lieu à une célébration quasi-familiale $»^{18}$. Forman, Jaroslav Papousek, Ivan Passer, Jiri Menzel, Vera Chytilova, Jan Nemec, Evald 
Schorm, Ladislav Helge et Vojtech Jasny. Leurs travaux représentent un "enregistrement", ou une "exposition", de la réalité quotidienne et contiennent des références à la tradition néoréaliste italienne et le cinéma-vérité français. Les principaux thèmes abordés traitent de la tension entre l'individu et la société avec une emphase sur les épisodes humoristiques en tant que source d'une certaine autodéfense ou résistance aux cruautés de la vie quotidienne. Les films de Menzel peuvent être distingués des autres du fait de son utilisation du film comme étant au service direct de la littérature. Il fait montre de certaines influences suréalistes que l'on retrouve dans l'œuvre d'Emir Kusturica, dont il était le professeur quand Kusturica, lui aussi, étudiait à Prague (1974-1978).

18 Kusturica a déclaré lors d'un interview qu'étudier à Prague, en terme de films visionnés, de livres lus, l'avait aidé à formuler sa relation envers sa patrie. Avant d'étudier à Prague, il pensait que sa propre expérience et l'endroit dont il venait étaient terribles, sans pertinence et primitifs. Les films représentaient, pour lui, un media par lequel on pouvait exprimer de grandes idées. La leçon de Prague a été on ne peut plus différente. Il a appris que l'universel pouvait être réalisé par le personnel et que le meilleur moyen d'avoir un large public était, en fait, qu'il présente les épreuves que toute personne doit traverser ${ }^{19}$.

19 D'autres membres des réalisateurs yougoslaves du Groupe de Prague, qui se trouvaient à Prague durant le "Printemps", en furent marqués de façons variées. Un certain nombre de films portent directement sur les événements en Tchécoslovaquie à cette époque et représentent une sorte de complainte pour les idéalistes de ce mouvement, notamment L'été trompeur de 1968 (Varljivo leto 68, 1984) de Paskaljević. De nombreux autres développent des thèmes qui sont très liés au noyau thématique des réalisateurs tchécoslovaques. L'impact direct des événements de cette période est difficile à appréhender, bien qu'il soit utile de rappeler que Rajko Grlić appartenait au comité de grève de l'université au moment de l'occupation soviétique ${ }^{20}$.

La créativité de la plupart des champs fut supprimée, suite à l'échec des réformes en face $d u$ courant politique plus conservateur. L'invasion soviétique de la Tchécoslovaquie à la suite du "Printemps de Prague" réintroduisit l'orthodoxie communiste dans tous les champs de la vie, période connue sous le nom de "long silence". L'industrie du cinéma, en général, et les réalisateurs de la Nouvelle vague, en particulier, furent victimes de la répression massive lors de la "normalisation", bien que dans la plupart des cas, ce ne fut pas avant 1970-1971, quand les réalisateurs yougoslaves passaient leurs examens. Les films de cette période furent ré-évalués, ce dont la citation de Ludvik Toman, directeur littéraire aux studios Barrandov rend le mieux compte. Ses critiques en 1972 rappellent étrangement celles émises à l'encontre du Nouveau cinéma yougoslave à la même époque : " nous sommes intéressés par l'art qui rejette et critique le scepticisme, les sentiments d'aliénation, le désespoir, la sexualité inconsidérée, l'individualisme bourgeois égoïste. Nous voulons soutenir par nos films ces propriétés qui renforcent notre société et non pas celles qui l'affaiblissent ${ }^{21} »$.

\section{Les films du Groupe de Prague}

21 À la fin des années 1970 et au début des années 1980, à la suite de la répression à l'encontre du Nouveau cinéma et une "vague rouge" à l'éloge plutôt cru de films de guerre pro-régime, il y eut une renaissance de réalisateurs plus recherchés en 
Yougoslavie, avec toute une série de films obtenant aussi bien un succès populaire que critique, aussi bien dans le pays qu'à l'étranger. On peut attribuer la contribution majeure à cette renaissance du cinéma au Groupe de Prague. Ce groupe a utilisé la même approche méthodologique de la réalisation, en traitant des sujets plutôt similaires avec des instruments stylistiques similaires. Notamment, ils partageaient une affinité pour l'expérimentation du film en tant que mode de communication audiovisuelle. Cette approche méthodologique unique qu'utilisaient les membres du Groupe de Prague est synthétisée par la notion de "populisme méthodologique élitiste " développée par Turkovićn ${ }^{2}$. Elle suggère une approche artistique complexe dans laquelle un d'auteur, pour la réalisation de son œuvre de caractère élitiste, emploie également des éléments populaires afin d'obtenir une audience plus large. Turković considère que Occupation en 26 scènes (Okupacija u 26 slika, 1978) de Zafranović, La couronne mortuaire de Petria (Petrijin venac, 1980) de Karanović, La mélodie hante mes songes (Samo jednom se ljubi, 1981) de Grlić, et Te souviens-tu de Dolly Bell ? (Sjećaš li se Dolly Bell ?, 1981) de Kusturica, représentent les exemples les plus marquants de cette approche ${ }^{23}$.

Un autre courant de populisme méthodologique élitiste tente de marier des éléments d'élitisme d'auteur et de populisme folklorique. C'est ce courant qui domine dans la plus grande partie de l'œuvre de Kusturica. Selon Turković, les " populistes honnêtes » sont des auteurs / réalisateurs professionnels qui apprécient la tradition urbainepopulaire et ne dressent pas de frontière en termes de valeurs entre l'élitisme, en haut, et le populaire, en bas $^{24}$. Cette construction complexe n'est pertinente que si l'on se souvient que la Yougoslavie a connu une industrialisation rapide dans l'aprèsDeuxième Guerre mondiale, tout en demeurant divisée entre population urbaine et rurale. Tous les réalisateurs sont des citadins et célèbrent la vie urbaine dans leurs films, ce qui constitue un appel direct à un large public lui aussi concerné par une nouvelle sensibilité urbaine ${ }^{25}$, qui dévoilait la ville dans tous ses aspects, aussi bien négatifs que positifs.

Parfois, la méthodologie semble influencer les thèmes traités, et parfois cela semble être l'inverse. Au niveau stylistique, ce qui apparaît le plus souvent, ce sont des personnages présentés de manière satirique, absurde et tragicomique. La seule exception est l'œuvre de Zafranović qui emploie un esthétisme quasi-élitiste. On remarque la tendance à l'expérimentation dans ses films où les acteurs sont des amateurs ou des personnes jouant leur propre rôle, «tentant de découvrir nos espaces authentiques par des expressions cinématographiques réduites et crues ${ }^{26}$.

L'amplitude réelle des relations entre les membres du Groupe de Prague est difficile à déterminer au travers des interviews publiés. Bien qu'aucun d'entre eux ne fasse référence à cette étiquette, de telle sorte qu'elle leur est imposée plutôt que souhaitée, il existe certainement de fortes relations. Karanović et Grlić, par exemple, ont travaillé ensemble sur six projets, tour à tour réalisateur et scénariste. De plus, ils ont tous tendance à utiliser le même groupe d'acteurs / actrices, notamment Mirjana Karanović, Predrag (« Miki ») Manojlović et Slavko Stimac.

\section{Méta-thèmes dans le travail du Groupe de Prague}

Les membres du Groupe de Prague ont traité une large variété de thèmes, de périodes, en utilisant des styles variés. On peut néanmoins dégager un certain noyau thématique, 
parfois camoufflé, une sorte de méta-narration. Ces thèmes, qui font référence aux relations sociales contemporaines et aux expériences du passé récent, représentent le lien commun qui unit les membres du groupe et aident à mettre en évidence la résonance des films avec la majeure partie de la société yougoslave des années 1980. Ces méta-thèmes ont toujours été présentés de manière satirique et ironique, avec un humour noir et une fin plus ou moins pessimiste. On peut en dégager cinq.

\section{1 - Rencontre avec le nouveau chef : les idéaux de la révolution trahis}

Les participants à la révolution socialiste authentique sont décrits comme affrontant une réalité dans laquelle ils prennent conscience que les idéaux pour lesquels ils se sont battus sont pervertis et manipulés par des apparatchiks dont le seul but est de maintenir le statu-quo et leur propre position dans le système. Ces idéalistes deviennent de plus en plus désenchantés et se désintègrent psychologiquement et émotionnellement. Bien que ce thème soit censé se dérouler dans l'immédiat aprèsguerre, au lendemain de la victoire des Partisans, le complot et les événements peuvent facilement, avec un peu d'imagination de la part du public, être transposés dans le contexte des années 1980 .

L'exemple majeur de ce thème est La mélodie hante mes songes de Rajko Grlić. Le film est basé sur un journal des événements au lendemain de la Deuxième Guerre mondiale. Il rend compte de la désintégration psychologique d'un héros de guerre, jeune Partisan idéaliste, au bagage culturel limité, qui tombe amoureux et se marie avec une ballerine de la classe moyenne. Entrant dans une famille "bourgeoise", il se retrouve au milieu de contradictions propres à la société. Les idées révolutionnaires pour lesquelles Tomislav s'est battu sont à l'opposé du mode de vie que sa femme tient à conserver. De plus, elle ne l'appelle pas par son nom, mais par son diminutif, de consonance occidentale “Tomi”. Il tente de réconcilier les problèmes qu'il rencontre dans sa vie privée avec les charges de dirigeant d'une communauté socialiste en formation au sein d'un petit village. Il doit faire face, de plus en plus, à la condamnation de ses camarades du Parti. Le monde intérieur de Tomislav s'écroule en même temps qu'il comprend que ses idéaux ne peuvent s'accommoder avec la dure réalité sans être déformés. Ses tentatives de faire concilier ces éléments divergents, mais parties intégrantes de sa réalité personnelle et culturelle échouant, il devient indésirable aux yeux des autorités et est même emprisonné.

\section{2 - La nouvelle classe : élite contre classe ouvrière}

C'est une autre critique clairement exprimée des relations sociales existant dans la société yougoslave des années 1980. Les membres de l'élite, la "nouvelle classe » de Djilas $^{27}$, sont dépeints comme extrêmement ambitieux et prêts à mettre en œuvre tout ce qui est nécessaire pour parvenir à leurs fins. La richesse, ou le capital économique, ne joue qu'un rôle marginal dans ce processus ; c'est le capital culturel et universitaire associé aux relations personnelles et la connaissance des bonnes personnes aux bonnes places qui sont perçus comme étant les éléments les plus importants. À l'opposé, dans de nombreux films, les membres de la classe ouvrière croient encore aux idées de la révolution socialiste, bien qu'elles soient déformées pour s'adapter aux intérêts de ceux qui détiennent les ressources et le pouvoir. 

souviens-tu de Dolly Bell ? de Kusturica. Bravo maestro décrit la tension qui existe entre l'ambition individuelle et les contraintes de la réalité politique. Il traite d'un jeune compositeur qui s'allie au pouvoir afin de travailler au grand jour. Il s'agit de la description d'une pratique de plus en plus utilisée par les Yougoslaves à cette époque, qui faisaient usage de contacts personnels afin de pallier aux dysfonctionnements évidents du système d'autogestion socialiste. La critique de Grli\} de la pratique de ses concitoyens est atténuée par la fin du film : le talent du compositeur diminue au fur et à mesure que sa gloire augmente.

Te souviens-tu de Dolly Bell ? se déroule dans la proche banlieue de Sarajevo à la fin des années 1950. Les principaux personnages sont un jeune homme, Dino, issu d'une famille ouvrière, au début de la puberté, et son père, malade. Son père conserve son idéalisme en dépit du fait que le seul obstacle au traitement de sa maladie est le manque de ressources. Il a transmis son idéalisme à son fils qui, puisque l'environnement dans lequel il vit offre peu, fait preuve d'autosuggestion et chante chaque soir dans le hangar : "chaque jour, d'une manière ou d'une autre, je fais des progrès ». Le film, par la présentation des phases les plus importantes dans la vie de chacun (le passage à l'âge adulte et le vieillissement) présente la réalité sociale des personnages comme un piège auquel il ne semble y avoir aucune issue, bien que l'humour noir de Kusturica associé au caractère empathique de ses personnages militent en faveur de l'existence d'une solution. La tragédie du père de Dino réside dans le fait que ses idéaux sont officiellement proclamés mais ne sont pas appliqués par ses contemporains, alors qu'il y croit encore. Dino tente d'éviter une telle tragédie en adoptant un autre genre de "religion", tout en ne rejetant pas son père. Kusturica catégorise son film comme une histoire d'amour, il est évident qu'il s'agit de bien plus, d'autant plus qu'il a depuis déclaré que chacun de ses films traitait de sujets contemporains et que ce film a été réalisé en 1981, un an après la mort de Tito.

\section{3 - Vivre à la marge}

L'un des tabous dans la société yougoslave des années 1980, qui déclarait l'égalité des chances et même l'égalité de tous, était la place de la population tsigane, et d'autres, vivant dans une pauvreté extrême. L'existence de ces deux problèmes, malgré la tentative de certains réalisateurs du Nouveau cinéma de les révéler au grand jour, a été couverte d'un voile de silence, ou déniée. Toutefois, les membres du Groupe de Prague les dépeignirent au public. Soulignant l'exploitation et la marginalisation des Tsiganes ainsi que l'immobilité sociale des individus manquant de ressources et de relations, ils émettaient clairement un avis politique.

L'exemple le plus célèbre est Le temps des gitans (Dom za vešanje, 1988) de Kusturica, une histoire d'amour entre deux Tsiganes, Perhan et Azra, entrecoupée de métaphores tirées de la mythologie tsigane et disposant d'une bande son extraordinaire signée par Goran Bregović. Les rêves des personnages principaux représentent une sorte de clef pour la lecture des métaphores et, en même temps, une rupture du mode narratif et l'introduction dans le monde surréel d'une communauté soudée en opposition à la “civilisation moderne". L'histoire surréaliste n'est pas sans points communs avec L'ange gardien (Andjeo Čuvar) de Paskaljević, d'autant plus que les deux sont basées sur le trafic réel d'enfants tsiganes entre la Yougoslavie et l'Italie. Plus important encore : Le temps des gitans est tourné en langue tsigane, avec des acteurs amateurs tsiganes.

Balkanologie, Vol. IV, $n^{\circ} 1$ | 2000 


\section{4 - L'héritage incomplet de la Deuxième Guerre mondiale}

Ce thème a souvent été traité sous la forme d'une vie idyllique dans les régions rurales soudainement détruite par la guérilla ou lentement érodée par les répercutions tardives de la guerre. Il semble que ce dernier aspect soit une critique formulée par les réalisateurs sur le manque de communication entre le centre et la périphérie de la société yougoslave, ainsi qu'entre les différentes communes et, évidemment, sur le sujet brûlant des attitudes différentes adoptées par les différents groupes vis-à-vis de la montée du fascisme.

L'occupation en 26 scènes de Zafranović qui dépeint poétiquement le mode de vie bourgeois du Dubrovnik d'avant guerre et l'occupation par les fascistes et leurs collaborateurs est un exemple frappant de ce thème. Zafranović tente non seulement de mettre en évidence le côté sombre du passé de la Croatie, mais également d'explorer la face sombre de la nature humaine. Les personnages sont décrits comme des piliers convenables de la société qui, dans certaines circonstances, deviennent des bêtes sauvages. La scène la plus controversée dure sept minutes et présente le massacre oustachi de prisonniers dans un bus.

\section{5 - La société malade : population contre bureaucratie}

L'implantation déformée des valeurs et des principes socialistes est perçue comme ayant un impact négatif sur la vie quotidienne des personnes ordinaires. Elles sont forcées de faire partie d'un système dont le fonctionnement ne parvient pas à prendre en compte leurs besoins réels. Les tentatives effectuées pour s'ajuster au système aboutissent souvent à un abandon cynique de tout idéal. Ceux qui cherchent à faire face, de front, au système, ou de le changer, finissent consignés aux marges de la société. La conclusion pessimiste, de nouveau en résonance avec le public des années 1980, est que la lutte humaine échoue à faire dévier le cercle vicieux de la bureaucratie socialiste.

$\mathrm{Au}$ contraire des autres thèmes, celui-ci peut se trouver en arrière plan dans la plupart des films. Mais le message est on ne peut plus clairement exprimé dans Variola vera (1982) de Marković. Il s'agit de l'histoire d'une épidémie due à une souche fatale de variole (variola vera) qui se répand dans le Belgrade des années 1970. L'hôpital est mis en quarantaine, l'incompétence du personnel hospitalier apparaît au grand jour, de telle sorte que les tensions au sein de l'hôpital représentent, clairement, une métaphore des tensions de la société yougoslave elle-même. Les autorités officielles tentent de contourner le problème en niant l'existence de l'épidémie, mais, lentement mais sûrement, elles doivent faire face à la réalité. À ce moment, il existe deux ordres sociaux, l'un dans l'hôpital isolé, extrêmement surveillé, et l'autre à l'extérieur où les autorités cherchent à ne révéler que des informations sélectionnées et apaiser les craintes de la population. Les deux mondes sont reliés par l'intermédiaire de l'expert médical qui, nous l'apprendrons plus tard, était immunisé au virus, et dont les vêtements protecteurs servaient à affirmer son autorité. Le film dispose de plusieurs niveaux de lecture. En fait, après avoir contracté le virus, un chef chirurgien, interprété par Radje Serbedžija, l'un des acteurs les plus populaires de cette époque, commença à faire le tour de l'hôpital, dans un état d'instabilité mentale, portant un imperméable, 
exactement comme Tito durant la guerre. Ce film offre une critique de la société qui tente de se présenter comme idéale.

Les thèmes que le Groupe de Prague n'a pas traité sont presque aussi important que les méta-thèmes. Tout comme pour le Nouveau cinéma, aucun des films réalisés par ce groupe ne se déroule dans la période précédant la Deuxième Guerre mondiale, que ce soit pendant la première Yougoslavie ou avant. En dépit de leurs critiques du régime, ils semblent partager implicitement la notion que l'histoire commence avec les Partisans. De plus, aucun des films du Groupe de Prague ne traite, en thème principal ou mineur, des relations entre les Républiques, ni de l'identité nationale ou ethnique. D'autres omissions ont plus à voir avec l'acceptation de certains tabous dans les discours publics. Il n'y pas de portrait explicitement critique de Tito, ni de la JNA, signifiant peut-être que ces deux "éléments" sont les seuls thèmes dont on ne peut pas parler ${ }^{28}$.

En ce qui concerne les paramètres socio-psychologiques, la manière dont ces films traitent des principales dichotomies (universalisme / particularisme, homogénéisation / différentiation, intégration / fragmentation ${ }^{29}$ ) est d'une importance particulière dans la compréhension de leur rôle dans la définition et le renforcement des identités particulières dans une Yougoslavie fédérale de plus en plus fragmentée.

\section{Universalisme - particularisme}

39 L'équilibre entre universalisme et particularisme a été réalisé au travers des thèmes choisis ainsi que de leur représentation. Situant leur sujet au niveau micro de la structure fédérale, c'est-à-dire dans les localités, à un niveau inférieur à celui des républiques, les réalisateurs ont évité la "républicanisation" des thèmes qu'ils ont abordés. Ils ont bien sûr réalisé leurs films à une époque où les débats politiques se déroulaient de plus en plus aux niveaux républicain et inter-républicain. Les films dressaient des portraits de réalités locales d'une manière sensible aussi bien positive que négative, reconnaissables par le public, sans en exagérer les traits.

Quelque chose dans l'intervalle de Karanović représente l'exemple type de cette opposition. La majeure partie du film se déroule à Belgrade, en Serbie, et on peut ressentir l'affection que porte Karanović à cette ville : certaines scènes ressemblent à une visite guidée bien que dépourvues d'explication touristique. Toutefois, au début du film, une brève scène se déroule à Dubrovnik, sur la côte croate, avec un portrait tout aussi affectueux de la ville. La suggestion évidente est que les personnages éprouvent les mêmes sentiments pour cette ville que celle dans laquelle ils vivent.

Le thème principal du film est la prise de conscience croissante des vraies réalités par une jeune journaliste américaine. Au début, sa connaissance de la Yougoslavie est très superficielle. Au fur et à mesure que le film se déroule, ses articles se font plus nombreux sur l'autogestion, le contexte de la carence en nourriture et en biens, les coupures d'électricité et la longue attente des professionnels pour obtenir leur appartement. La seule déclaration explicite sur la composition fédérale de la Yougoslavie est faite par cette journaliste qui dit: «vous ne pouvez même pas tuer votre Président : vous en avez huit ».

Dans ce film, comme dans de nombreux autres, les réalités particulières de la vie à Belgrade font corps avec des réalités quotidiennes de la vie dans la Yougoslavie fédérale de l'époque. En fait, en dépit des problèmes évidents que traverse la Yougoslavie, l'un des personnages principaux se définit comme "patriote". Le terme, dans le film, fait 
référence, sans ambiguïté, à une identité yougoslave et socialiste. À de nombreuses occasions, la journaliste américaine évoque les valeurs de la culture globale: le restaurant où on l'amène ressemble à un autre de New York, le burek qu'elle mange « ressemble à quelque chose du pays, mais avec une pâte différente ». Ces affirmations sont toutefois présentées humoristiquement et laissent transparaître qu'elle croit comprendre bien plus que ce n'est effectivement le cas.

\section{Homogénéisation - différentiation} opposition. Le film, qui se déroule dans un quartier de Sarajevo, donne une "saveur" de la communauté au travers, parmi d'autres scènes, la description d'une circoncision, faisant clairement du jeune homme un Musulman. Mais, les problèmes que rencontre cette famille sont les mêmes que ceux des autres membres de la société, de telle sorte qu'être Musulman est un fait culturel mais non politique, reflétant en soi les réalités urbaines de Sarajevo ${ }^{31}$. L'histoire se passe dans la période succédant à la rupture TitoStaline, quand le régime mettait tout en œuvre pour supprimer ce qui pouvait ressembler à des sympathies ou de l'idéologie staliniste. Cela a été si loin que ceux qui n'ont pas exprimé un enthousiasme rapide et ostensible pour la nouvelle situation ont été également victimes du régime. Kusturica présente ce thème hautement politique au travers des yeux et l'interprétation d'un enfant, ce qui aboutit à un amoindrissement de la gravité des faits et un manque de condamnation explicite des événements. Néanmoins, la réalité d'une situation tendue marquée par l'insécurité et le besoin constant de chercher des stratégies de survie est clairement montrée.

\section{Intégration - fragmentation}

Les films juxtaposent fréquemment la fragmentation des individus et du système luimême avec l'idéal de son potentiel d'intégration dans lequel plus personne ne croit, mais dont, en un sens, plusieurs films se lamentent de son absence. L'exemple de Variola vera de Marković révèle une division marquée entre "nous", gens ordinaires (peu importe que "nous" puissions être différents les uns des autres) et "eux", la bureaucratie du régime. La métaphore la plus forte de la possibilité de la survie du système est peut-être la scène où un jeune homme, amoureux d'une des docteurs en quarantaine, arrive à déjouer la surveillance policière et à entrer dans l'hôpital. Au milieu d'une épidémie mortelle, nous suivons l'évolution d'une histoire d'amour entre lui, l'idéaliste, et elle, la réaliste. Mais, quand il est mis fin à l'épidémie, il meurt d'une

Balkanologie, Vol. IV, $n^{\circ} 1$ | 2000 
cause inconnue. Cette scène, se déroulant à la fin du film, dénote d'un certain pessimisme quant au futur.

\section{Nationalisme et production culturelle} réalisateurs du Groupe de Prague ne portaient pas sur la montée du nationalisme en Yougoslavie dans les années 1980. En ce sens, ils diffèrent de nombreux autres domaines de la production culturelle, tel que la littérature, la poésie ou la musique rock : ces formes d'expression culturelle ont été, depuis le milieu des années 1980, de plus en plus impliquées dans l'affirmation de nationalismes particuliers. Une étude récente affirme qu'il n'y avait pas de "cinéma yougoslave méritant de porter ce nom dans les années $1980 »^{32}$, donnant à penser qu'il existait « une recrudescence de visions nationalistes ou ethniques ». Mon propos est que, bien qu'étant d'accord avec les facteurs relevés par cette étude, tels que l'amplification générale du nationalisme, l'accroissement du nationalisme dans les sphères culturelles, la chute des marchés, etc., l'analyse qui la soutend est fausse. Le Groupe de Prague produisait effectivement un "cinéma yougoslave" dans les années 1980 en dépit du fait que c'était une période de crise et d'immense fractionnalisation pour la Yougoslavie. Le Groupe de Prague était alors exceptionnel, non seulement pour la qualité de ses thèmes, mais aussi pour son échec à rendre compte du nationalisme, aussi bien en arrière-plan qu'explicitement dans ses films.

La décentralisation faisant suite à la constitution de 1974 créait les conditions nécessaires pour les intellectuels à l'occupation de l'espace structurel du nationalisme, les élites républicaines étant seulement concernées par leurs propres affaires. Les artisans culturels durant cette période ont surtout dû traiter avec leur propre république et non pas avec les agences fédérales. Ainsi, à la fin des années 1970, les productions culturelles étaient plus susceptibles d'être jugées en fonction du soutien ou de la menace qu'elles apportaient à chaque république, selon qu'elles portaient atteinte à leur "appartenance» ou «leur héritage culturel et traditionnel »" ${ }^{33}$. Un exemple crucial est donné par l'un des films réalisé par l'un des réalisateurs du Groupe de Prague, Quelque chose dans l'intervalle de Karanović. La question a été soulevée sur le fait qu'un des personnages principaux du film évoque une chanson comme étant «une belle chanson serbe $»^{34}$. Tout de suite après avoir remporté le prix du meilleur réalisateur à Pula, des journalistes macédoniens ont demandé à Karanović si la chanson en question n'était pas, en fait, une chanson macédonienne. Le réalisateur a répondu qu'il lui semblait qu'elle provenait du sud de la Serbie, mais qu'il pouvait se tromper. Le débat s'envenima au fil des colonnes des principaux quotidiens de l'époque, avec la participation de différents experts prouvant des choses différentes. De fait, le film ne fut pas autorisé en Macédoine, tant que le dialogue incriminé ne serait pas modifié ou supprimé, alors qu'il continuait d'être diffusé partout ailleurs ${ }^{35}$. Cet exemple montre clairement que les préoccupations des Républiques sont très différentes de celles des réalisateurs.

L'opus de Zafranović diffère de celui des autres membres du Groupe de Prague par sa préoccupation particulière d'un aspect du passé : les événements en Croatie durant la Deuxième Guerre mondiale. Son film Occupation en 26 scènes est peut-être le plus représentatif de son identification de la tâche d'un intellectuel: interroger le côté obscur de sa propre nation. Toutefois, son propos concernait une république au sein de

Balkanologie, Vol. IV, $n^{\circ} 1$ | 2000 
la Yougoslavie confédérale. Les réactions au film ont bien évidemment été variées : à Belgrade, en un mois, 200000 personnes avaient été le voir, et, selon International Herald Tribune, l'avaient perçu « comme comportant un fond de vérité »; à Zagreb, certains l'avaient encensé, d'autres avaient manifesté devant les cinémas, et d'autres encore avaient accusé Zafranović d'un manque d'équilibre en ne montrant que les crimes oustachis et pas les crimes tchetniks ${ }^{36}$. De fait, la plus grande réaction officielle contre un des membres du Groupe de Prague a été celle de la Croatie à l'encontre de Zafranović pour sa persévérance à exposer la collaboration fasciste au sein de cette république.

Bien qu'Emir Kusturica soit devenu plus tard une personnalité hautement controversée, les racines de cette controverse se trouvent, sous forme moins dramatique, à la fin des années 1980. Ainsi qu'il l'a dit, quand le script de Papa est en voyage d'affaires a été soumis au Conseil artistique, des membres ont exprimé leurs préoccupations à son sujet, considérant que les "purges" effectuées à la suite de la rupture entre Tito et Staline représentaient un sujet "sensible ${ }^{37}$. Expliquant son implication dans le groupe de rock de Sarajevo, Zabranjeno Pušenje (interdiction de fumer), qui faisait partie du sous-mouvement culturel "nouveau primitivisme", Kusturica déclara qu'il s'agissait d'une tentative de lier film et musique rock comme les modes d'expression alternatifs les plus importants du XX ${ }^{\text {ème }}$ siècle $^{38}$.

Le lien entre ses films et le projet culturel Zabranjeno Pušenje est important. L'un des desseins de ce mouvement était de présenter le retard culturel et politique apparent de la société yougoslave contemporaine, et la tension entre la ville et la campagne, les intellectuels et les masses, etc., de façon satirique. De plus, ce mouvement culturel jouait avec les stéréotypes de deux identités culturelles, balkanique et occidentale, ce qu'on remarque très bien dans le choix des pseudonymes des principaux «nouveaux primitifs », notamment Elvis J. Kurtović ${ }^{39}$.

51 Il se développait, clairement, des perspectives différentes au sein et entre les différentes républiques, la Slovénie et la Croatie se percevant de plus en plus occidentales et les autres républiques toujours balkaniques. Kusturica lui-même a été impliqué dans ce débat durant et après la réalisation de certaines scènes du Temps des Gitans en Slovénie, en 1988. À cette époque, bien sûr, la Slovénie disposait d'un mouvement culturel politique élaboré, dont le plus célèbre groupe de l'époque, Laibach (nom allemand de Ljubljana), qui, sous forme satyrique, utilisait souvent du symbolisme nazi dans leurs travaux ${ }^{40}$. Lorsque Branka Magaš a rencontré Kusturica à Ljubljana en 1988, il lui confia que durant la réalisation du Temps des Gitans, des habitants d'une petite ville avaient « attaqué les acteurs et l'équipe, en criant des slogans anti-tsiganes et anti-bosniaques $»^{41}$. À cette époque, Kusturica avait exprimé ses préoccupations lors d'un interview à Nin, sur la stagnation économique qui s'accentuait, associée à un fossé qui se creusait davantage entre les régions développées et les régions sousdéveloppées, lui-même source d'une intolérance croissante entre les différents groupes. Ses propos avaient été interprétées comme qualifiant la Slovénie de fasciste ${ }^{42}$. En fait, ses propos en révèlent plus sur ses préoccupations de l'époque :

Le fait qu'un facteur d'intégration n'existe pas est la chose la plus décourageante. Il n'y a pas de cohésion. Grâce à ce film, j'ai voyagé à travers tout le pays et j'ai vu comme la différence est grande (...). Je parle de différences mentales, parce que l'économie est dans un tel état qu'il n'y a pas de place pour qu'on se réunisse, sauf pour l'exploitation ${ }^{43}$. 
52

$$
\begin{aligned}
& \text { d'un des plus grands groupes de l'époque, Bijelo Dugme (Bouton blanc) de Sarajevo, a } \\
& \text { continué de célébrer les idéaux du yougoslavisme dans sa musique, mais Bijelo Dugme } \\
& \text { introduisit des mélodies "ethnicisées", de telle sorte que certaines de leurs chansons } \\
& \text { ont pu être scandées dans les manifestations de soutien à Milošević. Dans un entretien } \\
& \text { avec P. Ramet, en 1989, Bregović déclarait: "ils finiront par faire la guerre. Ce sera } \\
& 1941 \text { encore une fois. Nous y allons, c'est certain. Vous pouvez le voir même en } \\
& \text { musique. Les Serbes et les Croates ont leurs propres chansons qui sont plus importantes } \\
& \text { pour eux que n'importe quelle chanson "yougoslave" " }{ }^{44} \text {. }
\end{aligned}
$$

\section{Conclusions}

Durant les années 1980, les membres du Groupe de Prague ont, en essence, présenté et défendu, à travers leurs films, l'image d'une Yougoslavie dé-idéalisée, à une époque où presque toutes les forces sociales, hormis l'armée pour des raisons qui lui sont propres, l'avaient abandonnée. Les raisons en sont multiples. On peut voir le Groupe comme une "communauté culturelle" particulière, qui indépendamment de sa position géographique, partageait les mêmes connaissances, engagements et vision. En termes bourdieusiens $s^{45}$, le groupe a créé un champ culturel relativement autonome, virtuellement immunisé aux politiques incrémentales de l'époque.

Leurs années de formation se sont déroulées à Prague, à l'école d'art cinématographique de la FAMU, durant la période la plus créatrice du cinéma tchécoslovaque qui a été réprimé suite à l'invasion soviétique dont la plupart des réalisateurs ont, certainement, été les témoins, et en ont vu les conséquences. De plus, c'est à cette époque que les films critiques du Nouveau cinéma yougoslave étaient diffusés. Les réalisateurs ont appris directement de chacun de ces deux mouvements et, indirectement, mais de manière tout aussi importante, du sort réservé à ceux qui critiquaient trop ouvertement le système, c'est-à-dire que la critique directe du système ne serait pas tolérée.

55 Le contexte politique de la Yougoslavie des années 1980, dans lequel ces réalisateurs ont achevé leurs films ne peut pas être seulement décrit par les catégories de "libéral » ou "répressif". Il est très important de se rappeler que cette période est celle où chacune des républiques est devenue de plus en plus préoccupée par ses propres priorités et ses propres problèmes plutôt que par ceux de la fédération. Néanmoins, un film devait pouvoir franchir les frontières des républiques, ne serait-ce que parce qu'il était plus coûteux à réaliser, par exemple, qu'un livre ou un disque. Même si les réalisateurs l'avaient voulu, il y aurait eu des raisons de marché pour qu'un film "paroissien" bosniaque ou monténégrin soit moins facile à monter qu'un film qui, une fois situé, possède une résonance au travers de la fédération. Le public des six Républiques, chacune possédant un bagage culturel et historique différent de celui des autres, partageait le même héritage du passé récent, de telle sorte que cet héritage est devenu, en fait, l'un des thèmes majeurs du Groupe de Prague. Les réalisateurs le formant étaient les seuls acteurs culturels à continuer de le faire.

Le cinéma, l'un des produits culturels les plus coûteux, dépendait de sources de financement multiples. Pour pouvoir faire appel aux studios situés dans les différentes Républiques, les réalisateurs devaient faire attention dans le choix de leurs thèmes, puisqu'ils ne pouvaient pas se les aliéner. Cette situation de fait était encore plus

Balkanologie, Vol. IV, $n^{\circ} 1$ | 2000 
prononcée quand on prend en compte l'existence de nombreuses petites productions cinématographiques. À la fin des années 1970, le financement du film était de plus en plus recherché par la combinaison de ressources provenant de différentes Républiques et la co-production de réalisations inter-républicaines. Le film devait de plus récupérer la majorité de ses ressources du box-office et des ventes à la télévision ${ }^{46}$. Le contexte international est également important. La Yougoslavie des années 1980 se trouvait dans une phase de récession économique, thème que l'on retrouve dans plusieurs films du Groupe de Prague. De plus en plus, la coproduction avec des compagnies étrangères était le seul moyen de s'assurer que le film pourrait être achevé. Le prix des compétitions internationales est également devenu important pour pouvoir pénétrer les marchés internationaux. Il est clair que la plupart des réalisateurs pensaient de plus en plus à la possibilité de travailler aux Etats-Unis et de faire des films en anglais. En fait, bien que ne constituant pas une coproduction, Quelque chose dans l'intervalle de Karanović, réalisé en 1982, est, en grande partie, en anglais.

Les raisons pour lesquelles le Groupe de Prague ne s'est pas engagé dans le nationalisme républicain sont complexes et variées, plutôt que simples et unidimensionnelles. Ce groupe de réalisateurs est lui-même dispersé dans sa connaissance de, et son travail académique sur, l'éclatement de la Yougoslavie. On trouve des traces de leurs travaux, les réactions qu'ils suscitent et leurs propres motivations dans tous les États "post-yougoslaves". Cet article représente une tentative de raviver, dans le sens de remettre ensemble, quelques unes de ces traces. Rassemblées, elles doivent suggérer que le travail des réalisateurs du Groupe de Prague, de la fin des années 1970 à la fin de la Yougoslavie, était une réussite politique majeure et esthétique. Une recherche sur ce groupe de réalisateurs, en tant que partie d'une étude plus nuancée sur le passé, est nécessaire, non seulement pour aider à comprendre le rôle des formes culturelles dans les périodes de crise et de fractionnement, mais également pour aider à dessiner un futur moins nationaliste.

\section{NOTES}

1. Robinson (Gerturde J.), Tito's Maverick Media, Illinois : University of Illinois Press, 1977.

2. Magaš (Branka), The Destruction of Yugoslavia, London : Verso, 1993.

3. Turković (Hrvoje), « Interview with the author ", May 1998.

4. Turković (Hrvoje), Filmska opredjeljenja, Zagreb : Cekade Turković, 1985, p. 28.

5. Liehm (Mira), Liehm (Antonín.), The Most Important Art : Soviet and Eastern European Film After 1945, Berkeley : University of California Press, 1977, p. 417.

6. In Jacobsen (Kurt), « Mysteries of the Organism : interview with Dušan Makavejev », Leftcurve, (19), 1995, p. 15.

7. Cook (David A.), A History of Narrative Film, London : W.W. Norton and Co Cook, 1990, p. 729.

8. Pavlowitch (Stevan K.), The Improbable Survivor: Yugoslavia and its problems 1918-1988, Columbus : Ohio State University Press, 1988; Thompson (Mark), A Paper House : the ending of Yugoslavia, London : Hutchinson Radius, 1992. 
9. Filipović (Stevan), Milivojević (Marko), « Yugoslav Cinema Between Two Wars 1945-1995 », Bradford Studies on South Eastern Europe, (3), 1997, p. 17.

10. In Jacobsen (Kurt), art.cit., 15.

11. Cook (David A.), op.cit., p. 732.

12. "Komunist Roundtable on Situation in Cinematography ", Tanjug, 21/02/73.

13. Ibid.

14. Anderson (R.), «Yugoslav Student's Film Brings Demands Teacher Be Punished ", New York Times, 26/01/73.

15. "Yugoslav Filmmaker Gets Higher Sentence », Tanjug, 19/12/73.

16. Liehm (Mira), Liehm (Antonín), op.cit., p. 292.

17. Hames (Peter), The Czechoslovak New Wave, University of California Press : Berkeley Hames, 1985 , p. 30.

18. Ibid., p. 34.

19. «Neka vrsta otrežnjenja », NIN, 22/05/88.

20. Grlić (Rajko), « Biography », Internet, (http://www.ev.net/makefilm/rajko02.htm), 1998.

21. Hames (Peter), op.cit., p. 258.

22. Turković (Hrvoje), op.cit., p. 32.

23. Ibid., p. 34.

24. Ibid., p. 33.

25. Turković (Hrvoje), art.cit.

26. Vucković (M.), «Face and About Face : an interview with Srdjan Karanović », Moveast , (3), 1996, p. 163.

27. Djilas (Milovan), The New Class, New York : Harcourt Brace / Jovanovich, 1957.

28. Škrabalo (I.), « Interview with the author », May 1998.

29. Cf. McGrew (Anthony), «A Global Society?», in Hall (S.), Held (David), McGrew (Anthony), eds., Modernity and its Futures, Cambridge : Polity Press, 1992.

30. Ramet (Sabrina P.), Nationalism and Federalism in Yugoslavia1962-1991, Indiana University Press, 1992.

31. Bringa (Tone), Being Muslim the Bosnian Way, Princeton : Princeton University Press, 1995.

32. Filipović (Stevan), Milivojević (Marko), art.cit., p. 7.

33. Cf. Dević (Ana), «Ethnonationalism, Politics, and the Intellectuals : the case of Yugoslavia », International Journal of Politics, Culture and Society, 11 (3), 1998.

34. «Filmska varnica - bez sluha », Vjesnik, 27/08/83.

35. Ibid.

36. Dobbs (M.), « A New generation of Yugoslavs », International Herald Tribune, 03/01/79.

37. «Nisam pravio politički film », Borba, 28/05/86.

38. "Izvrnuo sam dušu », Vjesnik, 17/07/88.

39. Cf. Ramet (Sabrina P.), Balkan Babel: the disintegration of Yugoslavia from the death of Tito to ethnic war, Boulder : Westview Press, 1996.

40. Cf. Tomc (Gregor), « The Politics of Punk », in Benderley (Jill), Kraft (Evan), eds., Independent Slovenia : origins, movements, prospects, New York : St Martin's Press, 1994.

41. Magaš (Branka), op.cit., p. 134.

42. "Neka vrsta otrežnjenja » (art.cit.).

43. Ibid.

44. Ramet (Sabrina P.), «Whoever Doesn't Listen to this Song will Hear a Storm », in Ramet (Sabrina P.), ed., Rocking the State, Boulder : Westview Press, 1994.

45. Bourdieu (Pierre), "The Field of Cultural Production », in The Polity Reader in Cultural Theory, London : Polity Press, 1994.

46. Cook (David A.), op.cit. 


\section{AUTEUR}

JASMINA PAPA

Jasmina Papa est chercheur indépendant, à Zagreb. 